Oumarou Palou MADI ${ }^{1}$

Régis Peltier ${ }^{2}$

Oumarou Balarabé ${ }^{1}$

Mama NTOUPKA ${ }^{1}$

Nicole SIBELET ${ }^{3,4}$

${ }^{1} \mathrm{Irad}$

Programme Forêts et bois

Centre régional de recherche

agricole

BP 33, Maroua

Cameroun

${ }^{2}$ Cirad

UR B\&SEF

Campus international

de Baillarguet

F34398 Montpellier

France

${ }^{3}$ Cirad

UMR Innovation

73, rue Jean-François Breton

F34398 Montpellier

France

${ }^{4}$ Catie

7170 Cartago

Turrialba 30501

Costa Rica

\section{Abandon ou extension des plantations d'acacias au Nord-Cameroun : tout dépendra du fonctionnement des filières gomme arabique}

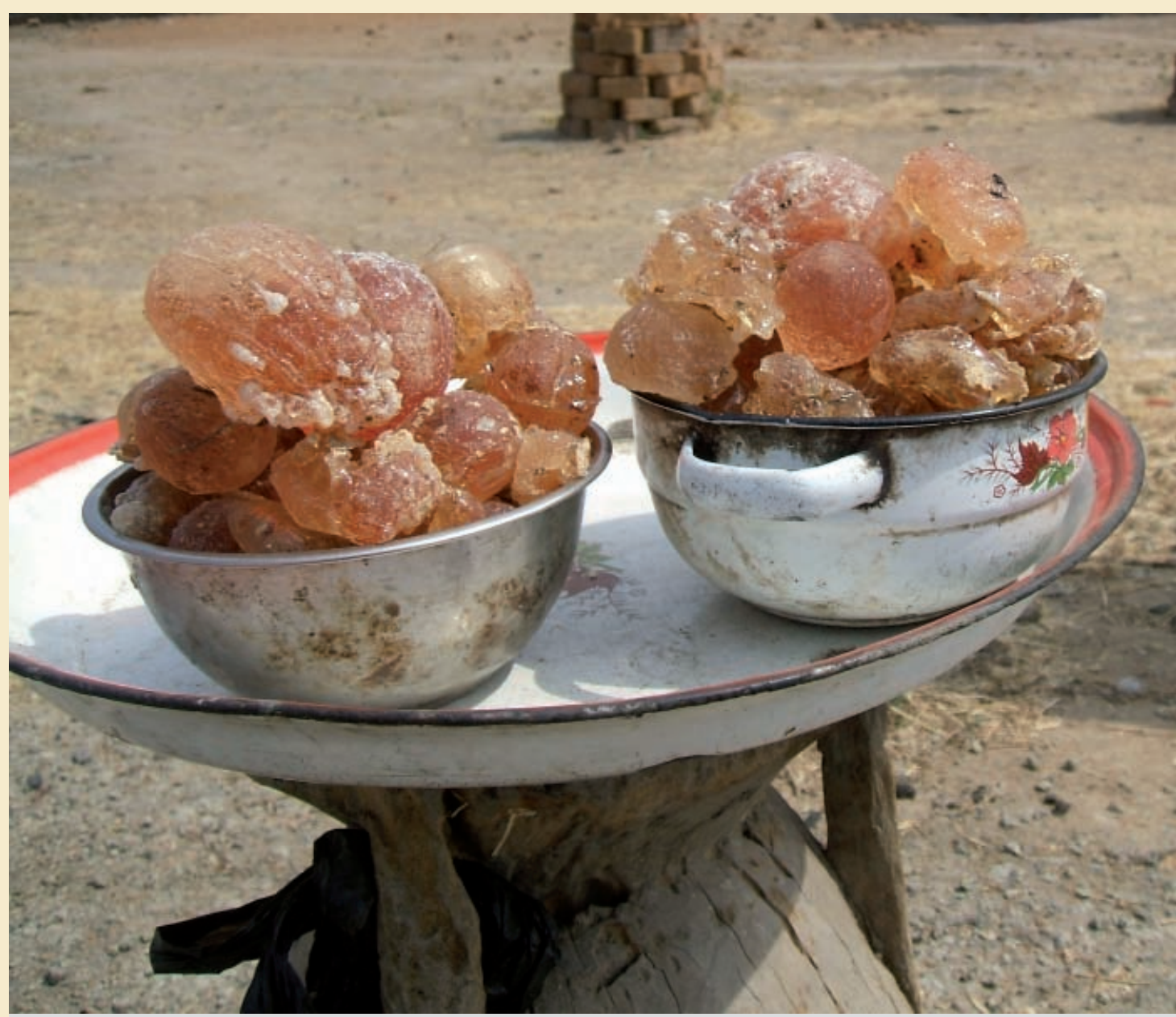

Koro de gomme, cuvette contenant $3 \mathrm{~kg}$, en vente sur un marché villageois. Photo O. Palou Madi. 


\section{RÉSUMÉ}

\section{ABANDON OU EXTENSION DES PLANTATIONS D'ACACIAS AU NORD- CAMEROUN : TOUT DÉPENDRA DU FONCTIONNEMENT DES FILIĖRES GOMME ARABIQUE}

Au Nord-Cameroun, plusieurs projets destinés à lutter contre la pauvreté et la dégradation de l'environnement ont encadré la mise en place de milliers de petites plantations paysannes d'Acacia senegal, entre 1990 et 2006. Cependant, depuis 2007, la plupart de ces plantations ne sont guère entretenues et n’ont pas été saignées pour la collecte. Les superficies plantées baissent et la filière formelle de gomme dure, issue de ces plantations, périclite. En 2008, la présente étude a comparé les caractéristiques des filières illégale et légale. Les résultats montrent que l'essentiel des quantités prélevées provient des formations naturelles à Acacia seyal (produisant la gomme friable) et emprunte une filière informelle vers le Nigeria. La cueillette est surtout effectuée par des enfants et des femmes. Malgré le faible prix d'achat de la gomme, cette filière présente des avantages pour les vendeurs désargentés, tels que le préfinancement de la campagne, le paiement au comptant et la multiplication des points d'achat. Les planteurs d'Acacia senegal (produisant la gomme dure de qualité) constituent une population très différente. II s'agit en majorité d'hommes âgés, aisés, partenaires des projets. La filière officielle qu'ils empruntent est rigide, les points d'achat sont peu nombreux et éloignés des lieux de cueillette et les achats et paiements ne leur sont pas garantis. Cette filière est fortement taxée officiellement et officieusement, ce qui la rend peu concurrentielle vis-à-vis de l'exportation illégale et entraîne une mévente de la gomme. Le prix payé au producteur $(0,2$ à $0,5 \$$ $\mathrm{US} / \mathrm{kg}$ ) est très inférieur à celui du marché international (1,5 à 4,5 \$ US/kg). La filière officielle ne peut être performante que si elle adopte les méthodes d'achat de l'informelle. Si l'État et les projets veulent encourager la gestion durable des peuplements d'acacias ou le reboisement, ils doivent sécuriser le foncier, réduire taxes et écueils commerciaux, tout en encourageant les producteurs à s'organiser et à se positionner sur des marchés plus rémunérateurs, de type commerce équitable ou biologique.

Mots-clés : Acacia senegal, Acacia seyal, gomme arabique, plantation paysanne, jachère enrichie, foresterie communautaire, commerce équitable, filière, Cameroun.

\section{ABSTRACT}

\section{SHOULD NORTH CAMEROON'S ACACIA PLANTATIONS BE ABANDONED OR EXTENDED? IT ALL DEPENDS ON DEVELOPMENT OF THE ARABIC GUM MARKET CHAIN}

From 1990 to 2006, thousands of small Acacia senegal plantations were established by farmers in northern Cameroon, under several projects aiming to reduce poverty and environmental degradation. Since 2007, however, maintenance has been poor in most of these plantations and few trees have been tapped. The planted area has declined and the formal market for hard gum from these plantations has plunged. In 2008 , this study compared the characteristics of the illegal and legal markets. Our results show that most of the gum is picked from natural Acacia seyal stands (which produce friable gum) and traded informally to the Nigerian market. The gum is mainly collected by women and children. Despite the low sale price of the gum, the informal market does have advantages for impoverished families, since it provides an advance for the next gum picking campaign, cash payments and a great many sales points. Those who plant Acacia senegal (which produced high-quality hard gum) make up a very different population. They are mostly men, elderly and well-off, who act as project partners. The formal market they supply is rigid, sales points are few and a long way from gum harvesting areas, and neither sales nor payments are guaranteed. This sector is heavily taxed, officially or not, so that it cannot compete with illegal exports and gum sales are slumping. The price paid to producers ( 0.2 to $0.5 \$ \mathrm{US} / \mathrm{kg}$ ) is much lower than on the international market (1.5 to $4.5 \$ \mathrm{US} / \mathrm{kg}$ ). The official market cannot operate effectively unless it adopt the trading practices used in the unofficial market. To encourage sustainable management of acacia stands, the State and the projects need to make land tenure secure and reduce taxes and other obstacles to trade while also encouraging producers to form organised groups that can take up a position on more rewarding markets of the fair trade or organic type.

Keywords: Acacia senegal, Acacia seyal, arabic gum, peasant plantations, improved fallows, community forestry, fair trade, market chain, Cameroon.

\section{RESUMEN}

\section{ABANDONO O AUMENTO DE PLANTACIONES DE ACACIAS EN EL NORTE DEL CAMERÚN: TODO DEPENDERÁ DEL FUNCIONAMIENTO DE LOS CIRCUITOS DE GOMA ARÁBIGA}

En el Norte del Camerún, varios proyectos destinados a luchar contra la pobreza y la degradación del entorno, enmarcaron el establecimiento de miles de pequeñas plantaciones campesinas de Acacia senegal entre 1990 y 2006 . Sin embargo, desde 2007, la mayoría de estas plantaciones carecen prácticamente de mantenimiento y no se efectúa el sangrado para la recolección. Las áreas plantadas disminuyen y el circuito formal de goma dura procedente de estas plantaciones decae. En el 2008, el presente estudio comparó las características de los circuitos ilegales y legales. Los resultados muestran que la mayoría de la goma extraída proviene de formaciones naturales de Acacia seyal (que produce goma, de consistencia friable) y sigue un circuito informal hacia Nigeria. Las tareas de recolección recaen sobre todo en niños y mujeres. A pesar del bajo precio de compra de la goma, este circuito presenta ventajas para los vendedores sin dinero, como la prefinanciación de la campaña, el pago al contado y la multiplicación de puntos de compra. Los cultivadores de Acacia senegal (que produce la goma dura de calidad) representan una población muy distinta. Se trata fundamentalmente de hombres mayores, acomodados y que participan como socios en los proyectos. El circuito oficial por el que transitan es rígido, los puntos de compra son escasos y están alejados de los lugares de recolección y, además, no se les garantizan las compras y pagos. Este circuito está fuertemente gravado, oficial y oficiosamente, lo que lo convierte en poco competitivo frente a la exportación ilegal y hace que la venta de goma decline. El precio pagado al productor (0.2 a $0.5 \$ \mathrm{US} / \mathrm{kg}$ ) es muy inferior al del mercado internacional (1.5 a 4.5 \$US/kg). El circuito oficial sólo puede ser eficiente si adopta los métodos de compra del informal. Si el Estado y los proyectos quieren fomentar el manejo sostenible de los rodales de acacias o la reforestación, deben garantizar la seguridad en la tenencia de tierras y reducir impuestos y trabas comerciales, a la vez que se impulsa a los productores a organizarse e insertarse en mercados más lucrativos como los del comercio justo u orgánico.

Palabras clave: Acacia senegal, Acacia seyal, goma arábiga, plantaciones campesinas, barbechos enriquecidos, silvicultura comunitaria, comercio justo, circuito, Camerún. 


\section{Contexte}

\section{Des plantations paysannes de gommier laissées à l'abandon}

Depuis les années 1980, les organisations gouvernementales et de nombreux projets, qui voulaient lutter contre la pauvreté et la dégradation de l'environnement, ont tenté d'instaurer une gestion durable des peuplements naturels d'acacias gommiers ou de susciter la création de plantations d'État ou communautaires au Nord-Cameroun. La recherche a montré que des plantations d'Acacia senegal peuvent avoir des productions de gomme de l'ordre de 150 kilogrammes par hectare et par an ( $\mathrm{kg} / \mathrm{ha} / \mathrm{an})$, en zone soudano-sahélienne du Cameroun (HARMAND et al., 1998). En outre, elles peuvent contribuer à régénérer des sols dégradés par la culture continue (HARMAND et al., 1997), sous forme de jachère améliorée, tout en fournissant du bois et du fourrage (Peltier, 1993). Pour ces deux raisons, les plantations de gommier ont été perçues par les organismes de développement du Cameroun (Sodecoton ${ }^{1}$ ) et par certains projets $\left(\mathrm{Dpgt}^{2}\right.$ et $\left.\mathrm{Esa}^{3}\right)$ comme une solution à encourager pour rénover la capacité des sols à produire du coton ou des céréales et pour diversifier les revenus des paysans (BERGER, LE COËNT, 2001). C'est ainsi que les projets Dpgt et Esa ont encadré la plantation d'environ 3000 parcelles d'un quart d'hectare d'Acacia senegal en milieu paysan, entre 1990 et 2006 (SOdECOTON-DPA/ESA, 2006 ; PALOU MAdI, 2007), dont environ $20 \%$ en zone Bénoué et $60 \%$ en pays Toupouri.

La plupart de ces projets ont également essayé d'organiser les filières de la gomme arabique ${ }^{4}$ au Cameroun, en vue d'en contrôler et d'en taxer les flux. En 2007, les visites de terrain ont permis de constater que la plupart de ces plantations étaient peu entretenues et ne présentaient pas de traces de saignée (PELTIER et al., 2009). D'autre part, les superficies plantées ont crû rapidement entre 1999 et 2003, pour baisser ensuite de 2003 à 2005 (figure 1). Il n'existe pas de données fiables pour la suite, mais rien n'indique une reprise significative des plantations.

\section{Une filière de la gomme de plantation qui n'arrive pas à se mettre en place}

L'idée couramment avancée pour expliquer le désintérêt des paysans pour la gestion et la saignée de leurs plantations est que non seulement les techniques ne sont maîtrisées que par une minorité d'entre eux mais que, surtout, la gomme produite ne trouve pas acquéreur.

Cela est une aberration économique car :

- les paysans ont d'importants besoins monétaires, surtout dans le contexte actuel de crise cotonnière ;

- ils ont d'importantes disponibilités en heures de travail en saison sèche, au moment de la récolte de la gomme ; même s'il est vrai qu'ils sont très occupés au moment de la saignée, qui coïncide avec la période de récolte de la plupart des cultures ;

\footnotetext{
${ }^{1}$ Société de développement du coton.

2 Projet Développement paysannal et gestion de terroirs.

${ }^{3}$ Projet Eau, Sol, Arbre.
}

${ }^{4}$ Il faut ici rappeler que, d'après les normes alimentaires de la Fao/Oms (Codex alimentarius), seules les gommes provenant de Acacia senegal et $A$. seyal peuvent être vendues sous l'appellation "Gomme arabique » (ou « Gomme d'acacia », code E 414).

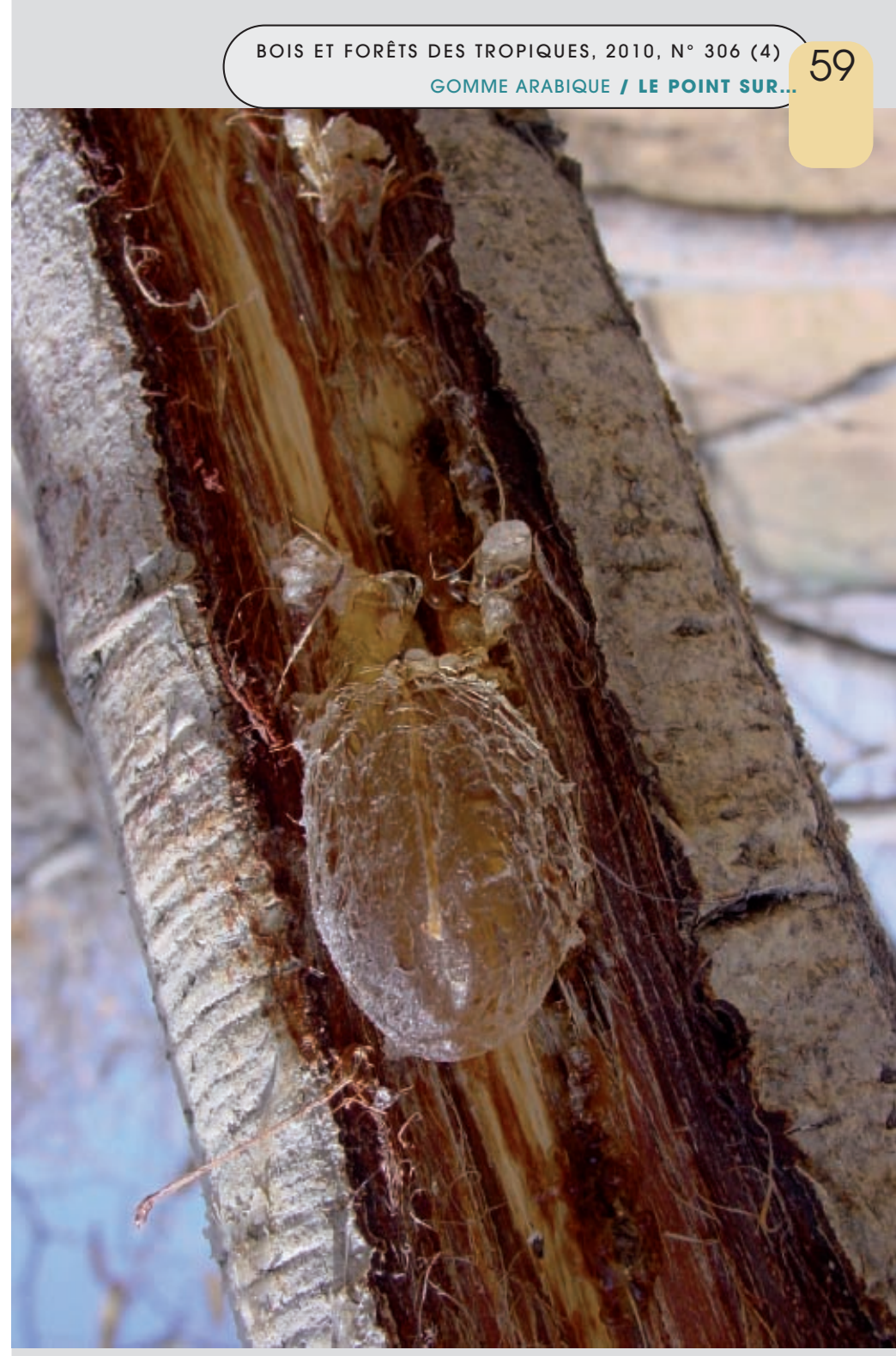

La recherche a montré que les plantations d'Acacia senegal peuvent avoir des productions de gomme de l'ordre de $150 \mathrm{~kg} / \mathrm{ha} / \mathrm{an}$, en zone soudano-sahélienne du Cameroun. Photo R. Peltier

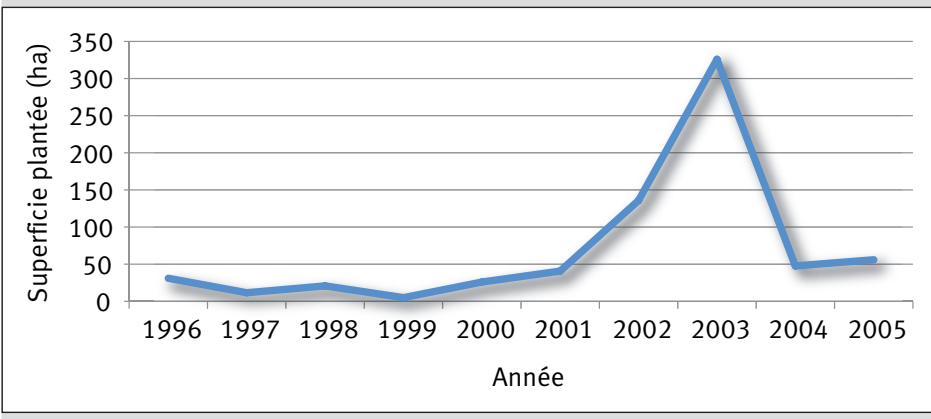

Figure 1.

Variation des surfaces plantées en Acacia senegal au NordCameroun entre 1996 et 2005.

Source : recensement des plantations et planteurs d'Acacia senegal (CONVENTION IRAD-SNV, 2006 ; SODECOTON-DPA-ESA, 2006). 
- la surface des terres dégradées est très importante et ceci depuis plusieurs décennies (TRIBOULET, 1993) ;

- les paysans ont un besoin de sécurisation foncière sur les terres en jachère que peut permettre la plantation (TEYSSIER, 2003) ;

- la demande mondiale de gomme est soutenue (MALLET et al., 2003) et n'est pas entièrement satisfaite depuis le début de la guerre civile au Soudan (le prix par tonne était en 2007 d'environ 4500 dollars américains (\$ US), alors qu'il se situait à 1500 \$ US en 2003).

Au Cameroun, la recherche et les projets ont impliqué, depuis 1990, le seul agent économique officiel habilité à acheter, à conditionner et à exporter la gomme, la société Cexpro-Sarl ${ }^{5}$. Cette entreprise achète la gomme cueillie dans les formations naturelles du Nord-Cameroun, à travers un réseau de collecteurs. Dans le cadre du projet Fao ${ }^{6}-\mathrm{Snv}^{7}$ d'organisation de la filière, la Cexpro a négocié avec l'Intergomcam ${ }^{8}$ (NјOMAHA, 2008), pour acheter la gomme triée d'Acacia senegal à un prix fixé annuellement d'un commun accord. Cela concernait en particulier la gomme produite par les planteurs, sur des plantations monospécifiques. En 2007, ce prix était de 600 francs CFA par kilogramme $\left(F C F A / \mathrm{kg}^{9}\right)$. Cependant, cette filière légale et officielle «formelle » tarde à se mettre en place, alors que la filière "informelle », illégale et ne payant pas de taxe, entre le Cameroun, le Niger, le Tchad et le Nigeria, existe depuis plus d'un demi-siècle et semble toujours très active.

De ce fait, la filière gomme camerounaise est une filière à trois circuits de commercialisation, dominée par la gomme récoltée dans les formations naturelles (figure 2).

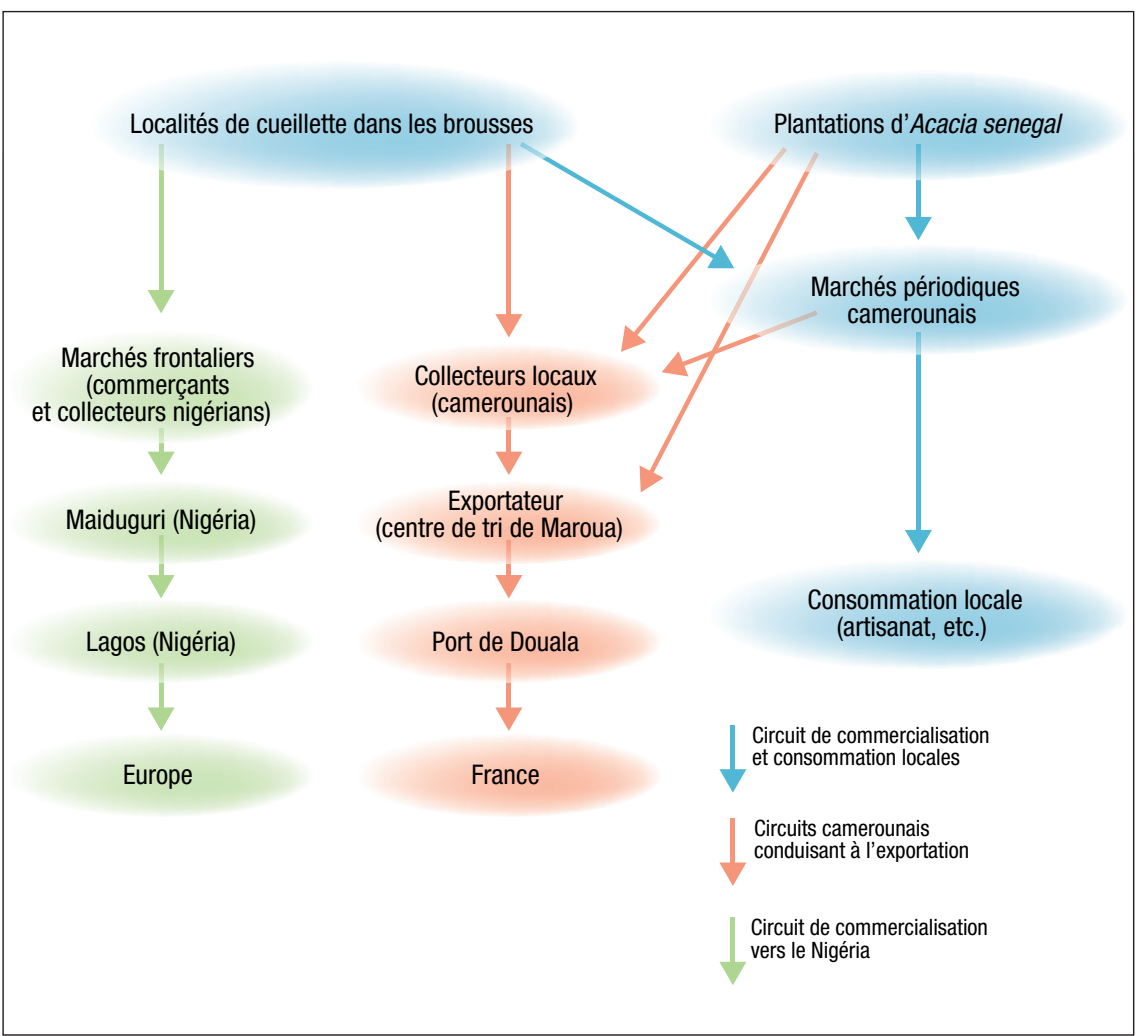

Figure 2.

Circuits de commercialisation de la gomme arabique camerounaise (MADI et al., 2002).

\section{Importance de la vente de gomme dans le revenu de certaines familles pauvres}

Concernant les revenus familiaux, NJOMAHA (2008) évaluait que l'activité de récolte de gomme au Nord-Cameroun contribuait en moyenne à hauteur de $37 \%$ au revenu monétaire familial des cueilleurs, constitué, par ailleurs, des produits de l'élevage (25\%), de ceux de l'agriculture (24\%) et de la vente du bois de feu et du petit commerce (14\%). La cueillette est effectuée en majorité par des enfants et des femmes, qui profitent d'une autre activité en brousse (ramassage du bois, garde des troupeaux, cueillette, etc.) pour compléter leurs revenus. Comme il s'agit de populations relativement pauvres ${ }^{10}$, malgré le faible revenu de cette activité, surtout rapportée au nombre d'heures de travail, elle est vitale pour eux.

NјомAнA estimait que la filière employait environ 50 pépiniéristes-producteurs de plants et 500 planteurs d'A. senegal, 1500 cueilleurs de gomme et 150 collecteurs. À l'Est du Niger, la gomme arrive en troisième position dans les revenus monétaires des familles. Au Sud-Est du Tchad, VARDON (1998) note que cette cueillette représente le principal revenu monétaire des familles de bergers transhumants, avec les graines de Balanites et les fruits de Tamarindus et de Ziziphus.

\section{Peu d'informations sur les cueilleurs et les collecteurs de gomme}

La nature des cueilleurs et des collecteurs camerounais et leurs relations n'étaient pas connues jusqu'à ce jour. Par contre, dans l'Est du Niger, DuHEM (2004) a montré que ce sont les enfants qui sont majoritaires parmi les cueilleurs (près de $50 \%$ ) par rapport aux femmes (34\%) (figure 3). La même étude souligne que $60 \%$ des collecteurs ont des cueilleurs-fournisseurs réguliers à qui ils passent des commandes et donnent des avances ; ils règlent quasiment toujours le solde au comptant. Près de $90 \%$ des collecteurs se déplacent au Nigeria pour écouler la gomme. Les quantités annuelles de gomme commercialisées sont proches de $1150 \mathrm{~kg}$ par collecteur. La gomme est achetée aux collecteurs par les grossistes nigériens entre 320 et 400 FCFA/kg (2004). Ces derniers la revendent entre 650 et 700 FCFA $/ \mathrm{kg}$. En moyenne, le prix payé au cueilleur représente près de $40 \%$ du prix de vente final de la gomme (figure 4). La marge du collecteur primaire représente $10 \%$ du prix final, celle du commerçant exportateur plus de $45 \%$.

\footnotetext{
${ }^{5}$ Compagnie commerciale pour l'exportation des produits forestiers spéciaux.

${ }^{6}$ Organisation des Nations unies pour l'alimentation et l'agriculture.

${ }^{7}$ Organisation néerlandaise de développement.

${ }^{8}$ Interprofession des producteurs de gomme du Cameroun.

91 franc CFA (FCFA) $=0,0021$ dollar (\$ US).

${ }^{10}$ Le produit intérieur brut du Cameroun est de 2199 dollars US par habitant.
} 


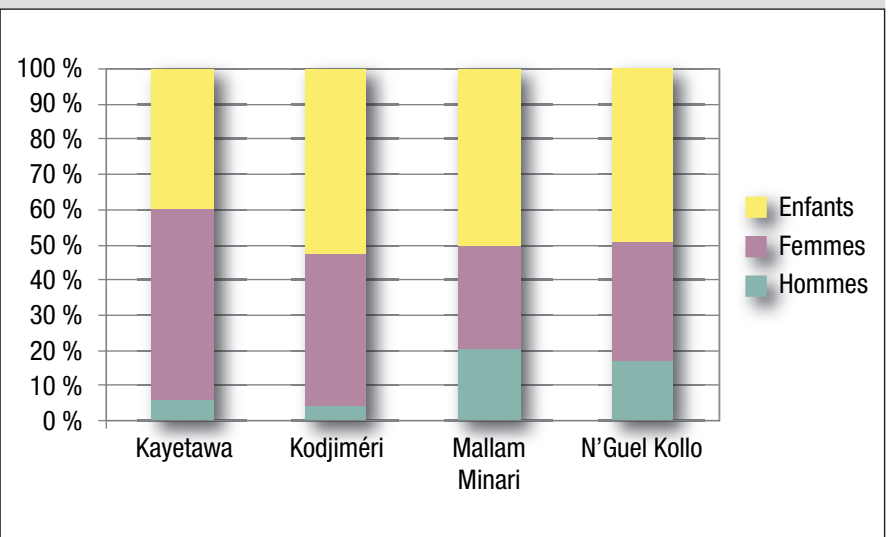

Figure 3.

Composition de la population de cueilleurs au Niger.

Sources : enquêtes filière gomme arabique, Pafn, 2004 (DUHEM, 2004).

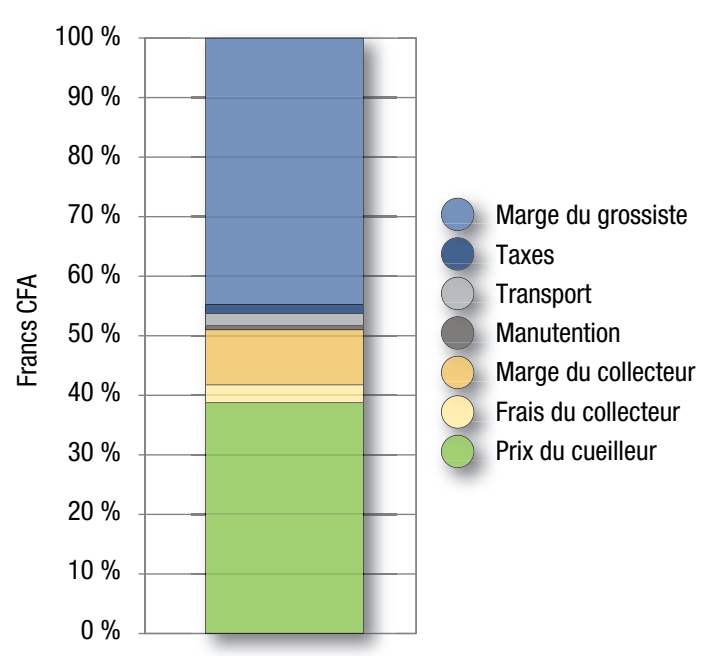

Figure 4.

Structure du prix de la gomme dans l'Est-Niger.

Sources : enquêtes filière gomme arabique, Pafn, mars 2004 (DUHEM, 2004).

\section{Cadre juridique et réglementaire de la filière de la gomme camerounaise}

En vertu de la loi 94-01 (Présidence de LA RépubliQue Du CAMERoun, 1994) et de son décret d'application 95/531/PM ${ }^{11}$, toutes les ressources forestières, à l'exception des forêts communales, communautaires et privées, des vergers, des plantations agricoles, des terres en jachère et des boisements, appartiennent à l'État. Toutefois, les populations dites « riveraines » (d'une forêt) voient reconnus leurs droits d'usage sur tous les produits forestiers en vue d'une utilisation «personnelle » (article 8).

Les dispositions relatives à la création de forêts communautaires tardent cependant, dans la région du NordCameroun, à être appliquées sur le terrain. Cette loi apporte une clarification sur les conditions d'exploitation des produits forestiers non ligneux (Pfnl) dont fait partie la gomme arabique (MADI et al., 2002). L'exploitation de tous les Pfnl dans les formations naturelles est soumise à la délivrance préalable d'un permis d'exploitation, mais ce cadre réglementaire (inapplicable car excessivement lourd et coûteux) est ignoré des populations paysannes.

\section{Une étude pour mieux comprendre les avantages de la filière informelle}

La présente étude a cherché à comprendre quelles étaient les caractéristiques de la filière informelle, de façon à tirer les enseignements qui pourraient permettre la redynamisation de la filière officielle, en particulier pour la gomme issue des plantations, le but visé étant de rendre rentable et durable la gestion des plantations.

Les résultats ont été comparés à ceux obtenus dans l'Est du Niger et au Tchad, dans des conditions écologiques et socio-économiques proches.

\footnotetext{
11 Premier Ministère.
}

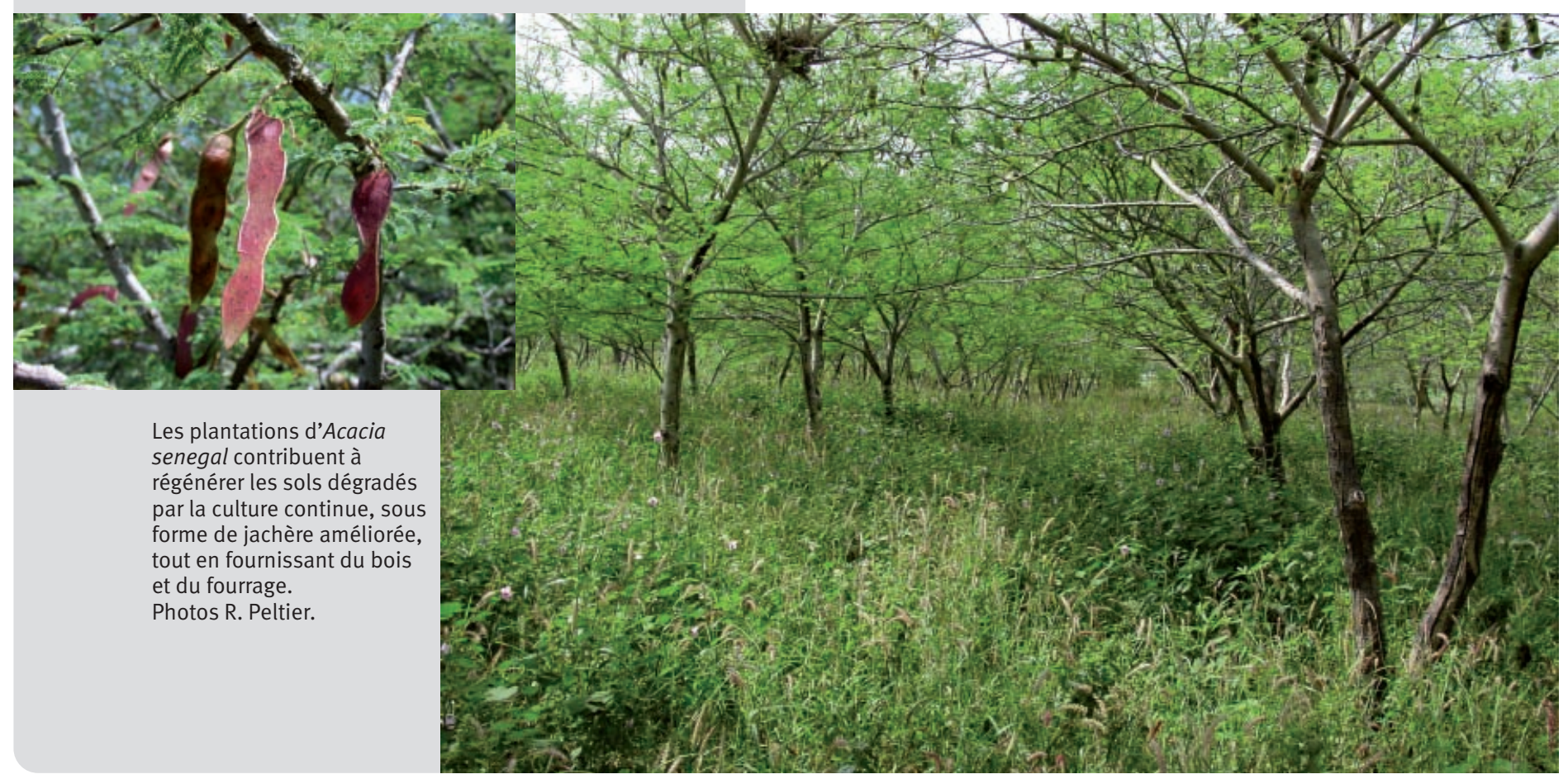




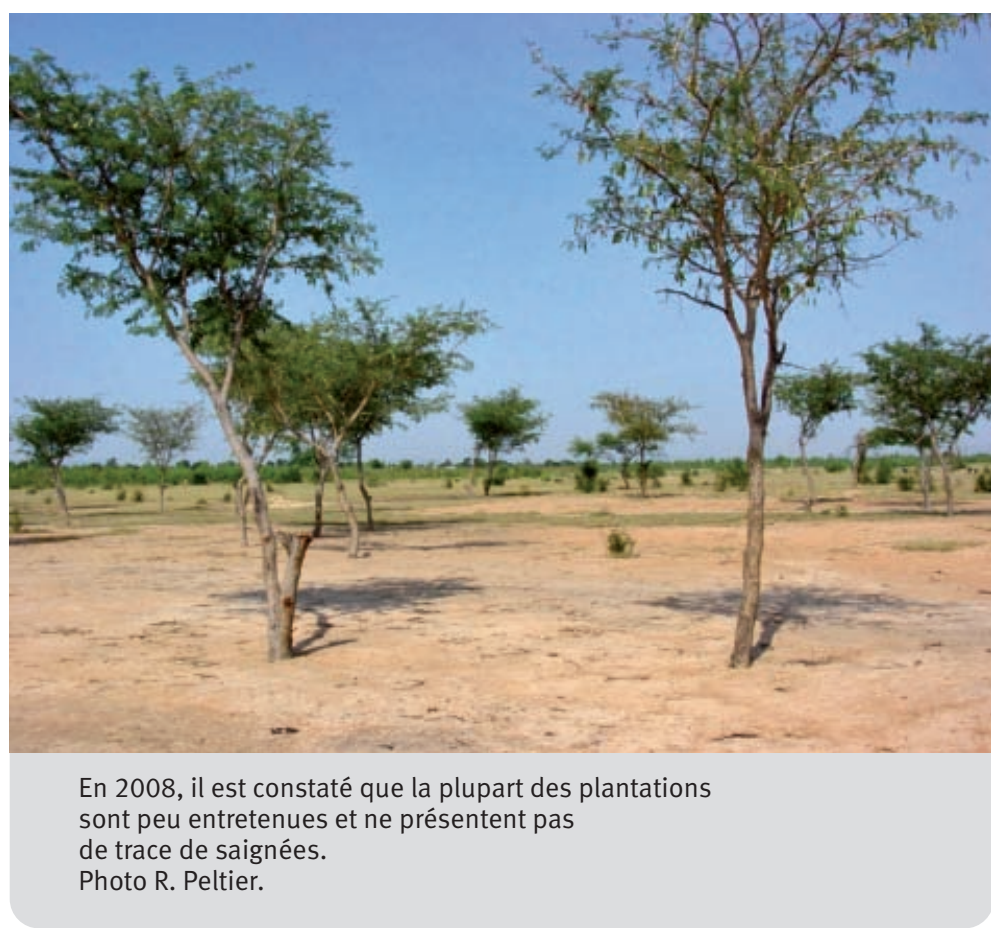

\section{Choix des sites d'étude}

Le tableau I donne la répartition des personnes enquêtées. D'autre part, dans chaque village enquêté, les lieux de récolte ont été visités (sans cartographie précise) et les espèces sur lesquelles était cueillie la gomme identifiées à l'aide d'une flore (ARBONNIER, 2000), sans inventaire. Le type de sol des lieux de prélèvement et la position topographique ont été notés.

Enfin, le cadre juridique et réglementaire a été précisé, par bibliographie, enquête auprès des services de l'État et mise en regard des pratiques réelles, dont faisaient état les cueilleurs, planteurs, collecteurs et acheteurs. La très grande majorité de ces enquêtes a eu lieu au Cameroun (pour des raisons d'autorisation et de sécurité); mais quelques observations et enquêtes informelles ont été menées au Nigeria, dans les marchés frontaliers.

${ }^{12}$ Centre d'étude de l'environnement et du développement au Cameroun.
Le choix des zones a été effectué sur la base de travaux antérieurs de zonage agricole (Dugué et al., 1994) et d'introduction des acacias gommiers (PALOU MADI, 2007) (figure 5). Deux zones d'étude en partie cotonnières ont été retenues pour l'étude sur les planteurs: Sud-Bénoué et pays Toupouri.

Pour la partie non cotonnière, la zone frontalière du Nigeria, de Kossa à Kousséri, a été retenue pour l'étude sur les cueilleurs. Aux sites retenus, il faut ajouter la ville de Maroua, chef-lieu de la région où se situent les échanges entre les acteurs intervenant dans la filière gomme, à travers le réseau interprofessionnel Intergomcam et le siège de l'exportateur Cexpro.

Le traitement des données, qui a été réalisé à l'aide du logiciel Microsoft Excel 2007, s'est limité à la statistique descriptive et à la construction des tableaux croisés dynamiques.

Tableau I.

Nombre d'enquêtés et périodes d'enquête.

\begin{tabular}{|c|c|c|c|c|c|c|}
\hline Type d'acteurs & $\begin{array}{c}\text { Zone } \\
\text { non cotonnière }\end{array}$ & $\begin{array}{c}\text { Zone } \\
\text { «Bec de canard }\end{array}$ & $\begin{array}{c}\text { Zone } \\
\text { Sud-Bénoué }\end{array}$ & $\begin{array}{l}\text { Nombre total } \\
\text { d'enquêtés }\end{array}$ & $\begin{array}{c}\text { Nombre } \\
\text { de femmes }\end{array}$ & $\begin{array}{l}\text { Périodes } \\
\text { d'exécution }\end{array}$ \\
\hline Planteurs & 5 & 20 & 15 & 40 & $2(5 \%)$ & Mars-avril 2008 \\
\hline Cueilleurs & 25 & 0 & 0 & 25 & $15(60 \%)$ & Juin 2008 \\
\hline Collecteurs & 8 & 0 & 0 & 8 & 0 & \\
\hline Pépiniéristes & 1 & 5 & 2 & 8 & 0 & Mars-avril 2008 \\
\hline $\begin{array}{l}\text { Structures privées } \\
\text { d'encadrement }\end{array}$ & 4 & 2 & 5 & 11 & - & Septembre 2008 \\
\hline Exportateur & - & - & - & 1 & - & Mai 2008 \\
\hline Total & 43 & 27 & 22 & 93 & 17 & \\
\hline
\end{tabular}




\section{Résultats}

\section{Principaux lieux de production et espèces d'acacias productrices de gomme}

Les enquêtes et les visites de terrain montrent que la zone non cotonnière, située le long de la frontière du Nigeria, de Kossa à Kousseri, est la zone par excellence des activités de cueillette de la gomme dans les formations naturelles.

Les exigences écologiques des diverses espèces productrices de gomme arabique sont différentes et se reflètent à travers leur distribution dans la zone d'étude. A. senegal, colonisant les sols sableux et les dunes, se retrouve en périphérie de l'ancienne cuvette du lac Tchad. A. seyal, plus indifférent aux conditions pédologiques, est présent dans presque tout le bassin du lac Tchad (avec une préférence pour les vertisols non durablement inondables).

Pour les autres espèces d'acacias productrices de gomme non classée « gomme arabique ", mais souvent mélangée par les cueilleurs à la gomme arabique, $A$. sieberiana, colonisant les sols limoneux et limonosableux, et $A$. polyacantha, plus fréquent sur les terrains alluviaux humides, se rencontrent autour du Parc national de Waza (Pnw).

\section{Composition de la population des cueilleurs camerounais}

La répartition des cueilleurs par classe d'âge et par genre (figure 6) montre qu'il s'agit en majorité d'adultes relativement jeunes, de 21 à 50 ans, dont une majorité de femmes, et de $10 \%$ d'enfants. Il s'agit en outre de populations à faible revenu ; mais ce paramètre n’a pas été évalué précisément.

Les migrants viennent surtout des villages dépourvus de peuplements naturels de gommiers et parfois du Nigeria. Ils résident dans les villages riverains du Pnw durant toute la période de cueillette.

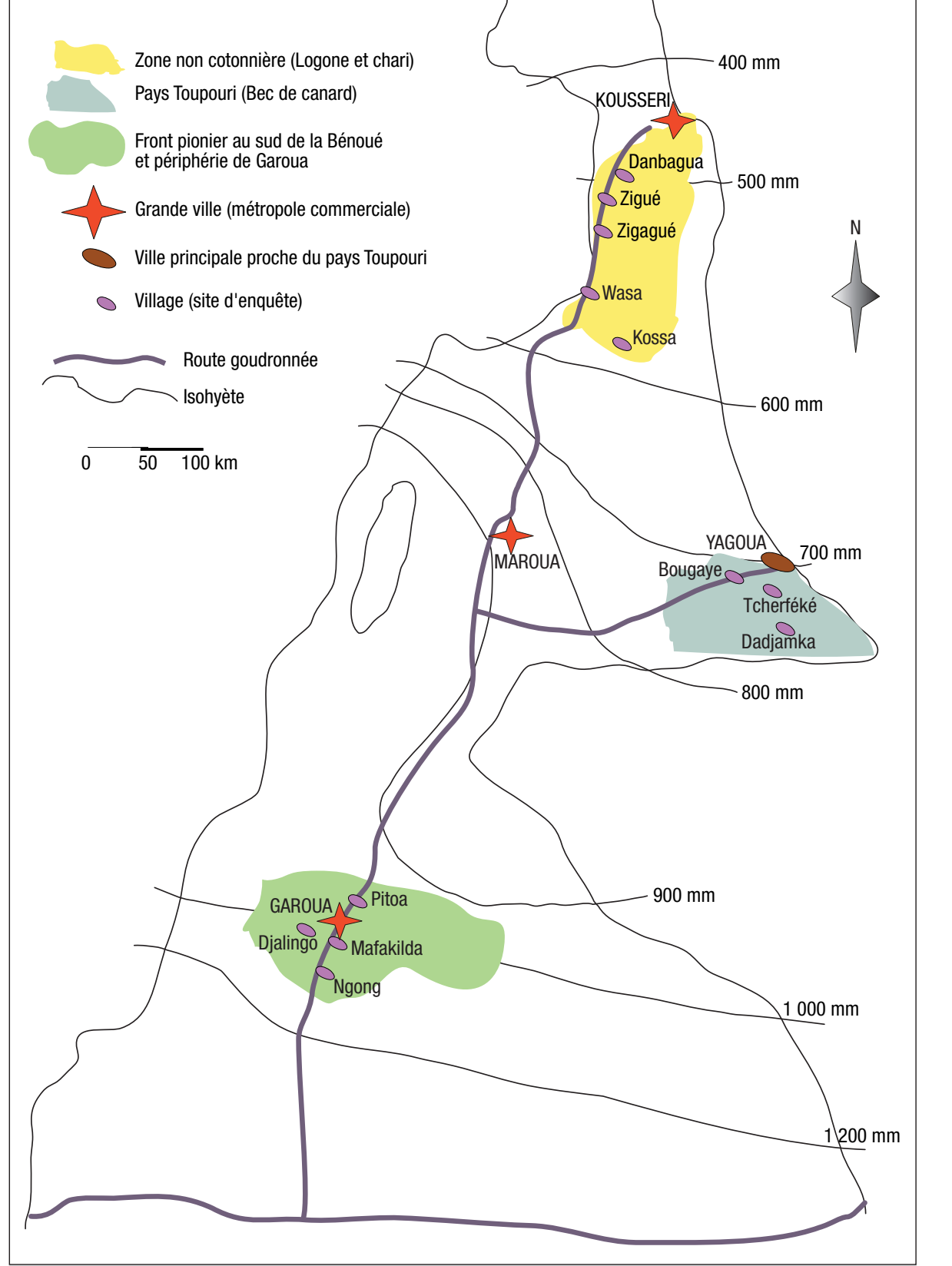

Figure 5.

Localisation des lieux d'enquête dans la zone soudanosahélienne du Cameroun.

\section{Pratiques de cueillette, dates et quantités}

En moyenne, sur la période de récolte 2007-2008, les cueilleurs de gomme se sont rendus en brousse cinq à six fois par semaine. D'après eux, le début de l'activité varie suivant les espèces d'acacias. $A$. polyacantha produit de la gomme quelques semaines (4-5) après la dernière pluie, tandis que la gomme d'A. senegal n'est disponible qu'à partir d'octobre et celle d'A. seyal et $A$. sieberiana qu'à partir de fin novembre. Le début de l'activité de cueillette de la gomme arabique est aussi influencé par les activités concurrentes dans le calendrier d'activités : culture du sorgho de contre-saison (muskuwaari) et pêche.

Les quantités cueillies annuellement, estimées par les enquêtés, varient d'un individu à l'autre, avec une moyenne proche de $5 \mathrm{~kg} /$ jour et de $500 \mathrm{~kg} /$ an (tableau II). Les quantités moyennes ramassées par les hommes sont nettement supérieures à celles des femmes et des enfants, à partir du mois de février, correspondant à la fin de la récolte du sorgho de contre-saison, muskuwaari. Au Cameroun, au cours de cette enquête, il n'a pas été rencontré de cueilleur qui pratique la saignée dans les formations naturelles. 


\section{Pratiques et prix de vente de la gomme par les cueilleurs}

L'unité de vente de gomme est le koro (petite cuvette émaillée qui, remplie de gomme, a un poids net d'environ $3 \mathrm{~kg}$ ). En moyenne, sur la campagne 2007-2008, tout au long des huit mois de cueillette, le prix d'un koro de gomme a varié de 210 à 1500 FCFA, soit de 70 à 500 FCFA/kg (figure 7).

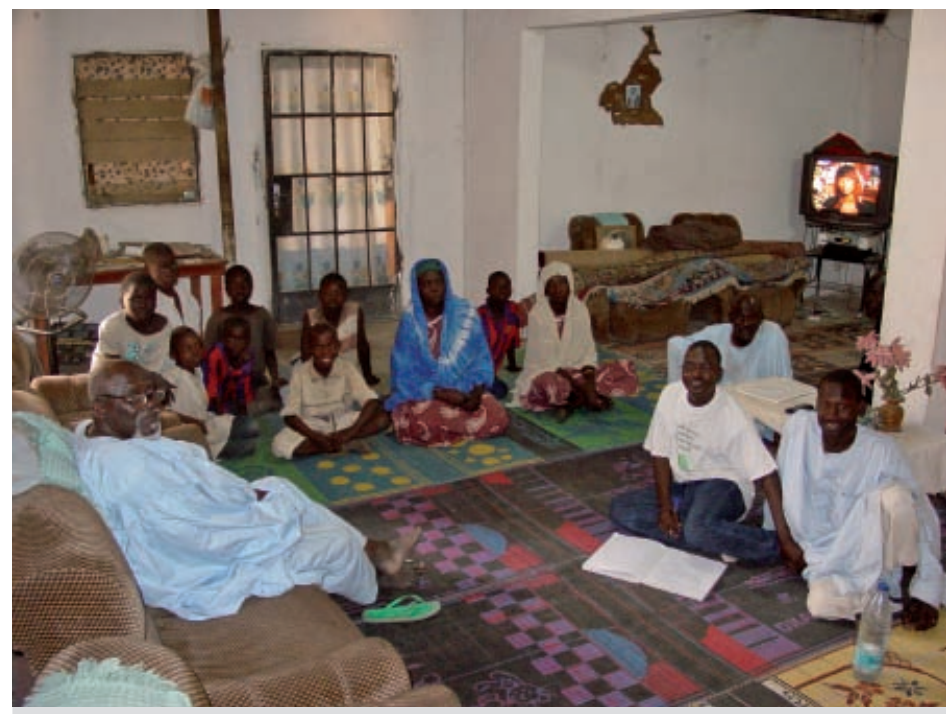

En 2008, des études de filière ont été menées au NordCameroun pour déterminer les pratiques réelles des cueilleurs et des acheteurs.

Photo R. Peltier.

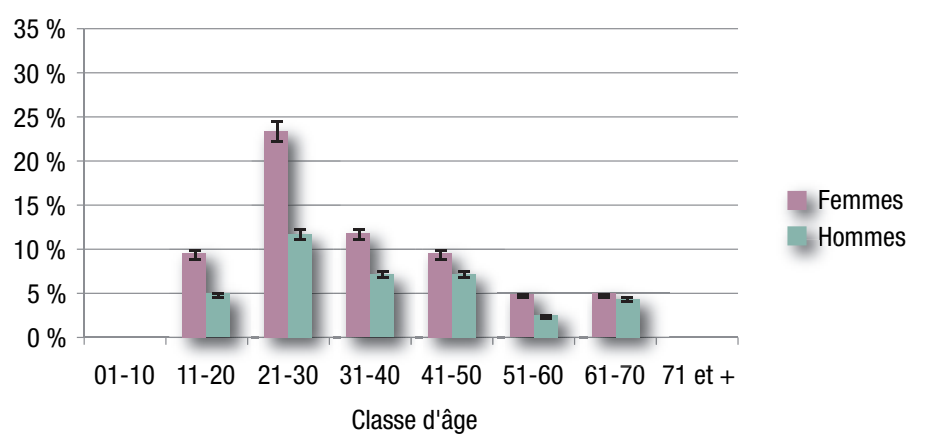

Figure 6.

Répartition de la population des cueilleurs de gomme camerounais par âge et par genre, en 2008.

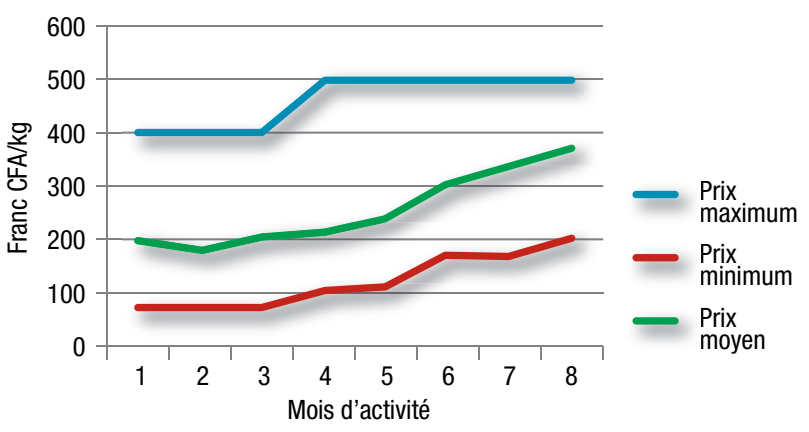

Figure 7.

Évolution annuelle du prix de la gomme par kilogramme au Nord-Cameroun (campagne de récolte 2007-2008).
Le prix maximum est atteint en fin de saison de cueillette et le minimum en début de campagne. En effet, dans ce dernier cas, la demande par les collecteurs est faible et l'accès à certains villages leur est encore difficile. Les cueilleurs sont des travailleurs occasionnels pauvres, souvent femmes et enfants, qui acceptent des prix bas. En fin de campagne, la gomme se fait rare en brousse, ceux des cueilleurs qui la vendent sont pour la plupart des hommes qui l'ont stockée, qui ont d'autres sources de revenus (pêche et bois de chauffe) et qui peuvent négocier. Le prix de 500 FCFA n'est atteint que pour ceux qui ont stocké leur produit au moins trois semaines après l'arrivée des pluies. Le prix moyen annuel, pour tout type de gomme et d'acheteur, au niveau cueilleur, est estimé à 260 FCFA $/ \mathrm{kg}$.

Ces prix de la gomme au niveau des cueilleurs villageois ne tiennent pas compte de la qualité (dure ou friable et degré d'impureté) car près de $62 \%$ des collecteurs interrogés ne font pas de catégorisation de la gomme lors de la collecte. De ce fait, les prix appliqués correspondent à ceux de la gomme friable d'A. seyal, qui sont les moins élevés sur le marché international. Les rares collecteurs qui catégorisent les gommes le font en fonction des espèces d'acacias sur lesquelles elles sont récoltées : gomme friable d'A. seyal et gommes dures d'A. polyacantha, $A$. sieberiana et $A$. senegal. Ces dernières, même si elles ne méritent pas toutes l'appellation « gomme arabique », sont en fait mélangées et vendues sous l'appellation locale, dont la traduction en français est " gomme d'A. senegal ».

\section{Détermination du prix d'achat au Cameroun par les acheteurs nigérians}

D’après les informateurs, les prix sur les marchés locaux sont déterminés par les opérateurs nigérians. En effet, ces derniers, bénéficiant de charges de commercialisation moins élevées (coûts de transport et de manutention plus faibles, non-paiement des impôts et taxes d'exploitation, etc.), ont la possibilité de proposer des prix plus élevés que l'acheteur officiel camerounais, afin de lui prendre des parts de marché. Les prix sont répercutés sur toute la filière jusqu'au niveau des villages de production, par le biais des commerçants et transporteurs. L'exportateur camerounais est alors obligé de s'aligner sur ces prix pour pouvoir s'approvisionner.

\section{Les collecteurs villageois, maillon indispensable de la chaîne}

Les collecteurs villageois sont des agriculteurs à temps partiel et des petits commerçants. Ils sont des intermédiaires d'achat des commerçants qui leur fournissent l'argent et les sacs d'emballage nécessaires à l'achat de la gomme. Les commerçants les rémunèrent par le versement d'une commission variant de 750 à 1250 FCFA par sac de 60 kg de gomme. Parmi les collecteurs, $36 \%$ affirment recevoir un préfinancement de la part des commerçants nigérians et/ou de l'exportateur camerounais, à hauteur d'environ 50000 FCFA par collecteur. Seuls les collecteurs membres du Gic Pdl ${ }^{13}$ de Waza (25\%) appartiennent à une organisation.

Les quantités de gomme collectées annuellement sont fonction des préfinancements reçus.

\footnotetext{
${ }^{13}$ Groupement d'intérêt communautaire, Plan de développement local.
} 
Tableau II.

Production de gomme chez les cueilleurs.

\begin{tabular}{|l|c|c|c|}
\hline Valeur & $\begin{array}{c}\text { Quantité cueillie } \\
(\mathrm{kg} / \text { jour })\end{array}$ & $\begin{array}{c}\text { Quantité par } \\
\text { vente }(\mathrm{kg})\end{array}$ & $\begin{array}{c}\text { Quantité cueillie } \\
\text { annuellement }(\mathrm{kg})\end{array}$ \\
\hline Maximum & 24 & 65 & 1620 \\
\hline Minimum & 0,75 & 6 & 40 \\
\hline Moyenne & 5 & 25 & 472 \\
\hline Ecarts-types & 5,3 & 16,61 & 3,88 \\
\hline
\end{tabular}

\section{Transport transfrontalier}

Le transport de la gomme permet, pour le circuit nigérian, la sécurisation du produit, par son passage de la frontière, avec, parfois, paiement de commissions illégales aux douaniers. Il se substitue alors aux taxes normalement dues. C'est ce qui explique son coût élevé (1 500 FCFA par sac de $60 \mathrm{~kg}$ pour seulement 80 kilomètres de parcours).

\section{Composition de la population des planteurs camerounais et pratique de récolte de la gomme}

Les plantations de gommiers sont principalement rencontrées dans la zone cotonnière, où les projets travaillant avec la Sodecoton les ont initiées. Les planteurs sont en majorité des hommes (95\%) adultes, âgés de 31 à 70 ans (figure 8). En outre, ils possèdent en majorité d'assez grandes surfaces (figure 9), sont supprimer relativement aisés par rapport à la moyenne régionale et sont en contact avec les agents de développement.
Bien que plus de la moitié des plantations soit âgée de cinq ans ou plus, au moment de l'enquête, et qu'elles puissent donc commencer à être saignées, seulement $20 \%$ des planteurs ont récolté au moins une fois leur gomme et l'ont vendue ; $3 \%$ à $18 \%$ des planteurs ont récolté de la gomme suite à des saignées artificielles pratiquées durant la campagne 20062007. Par manque de saignée, les productions sont très faibles : 5 à $10 \mathrm{~kg} / \mathrm{ha}$ en moyenne.

Les planteurs déclarent avoir planté en espérant des prix intéressants (de l'ordre de $600 \mathrm{FCFA} / \mathrm{kg}$ ) et la mise en place de points d'achat locaux. Mais ils préfèrent se consacrer à d'autres activités, plutôt que de vendre à un prix inférieur et de transporter la gomme vers des marchés lointains.

D’après le gestionnaire de Cexpro, la contribution de la gomme issue de plantation est très faible dans l'exportation ( $2 \%)$. Les ventes directes auprès de Cexpro sont rares et se font souvent par l'intermédiaire de l'Irad ${ }^{14}$.

Trente-cinq pour cent des planteurs n'ont aucune idée du prix et du lieu de vente et estiment que les points de stockage-vente créés ne sont pas fonctionnels, malgré les promesses des projets et de l'exportateur.

\section{Exportation officielle de la gomme}

D’après son gestionnaire, la société Cexpro exporte la gomme arabique camerounaise vers la France. Les quantités exportées sont restées sensiblement les mêmes depuis près de 10 ans, inférieures à 400 tonnes par an (t/an) ces trois dernières années. Celui-ci mentionne que le NordCameroun, à travers la ville de Kousséri, est une plaque tournante très importante pour la gomme arabique tchadienne, qui y transite pour le Nigeria et pour son exportation vers l'Europe, via le port de Douala.

${ }^{14}$ Institut de recherche agricole pour le développement du Cameroun.

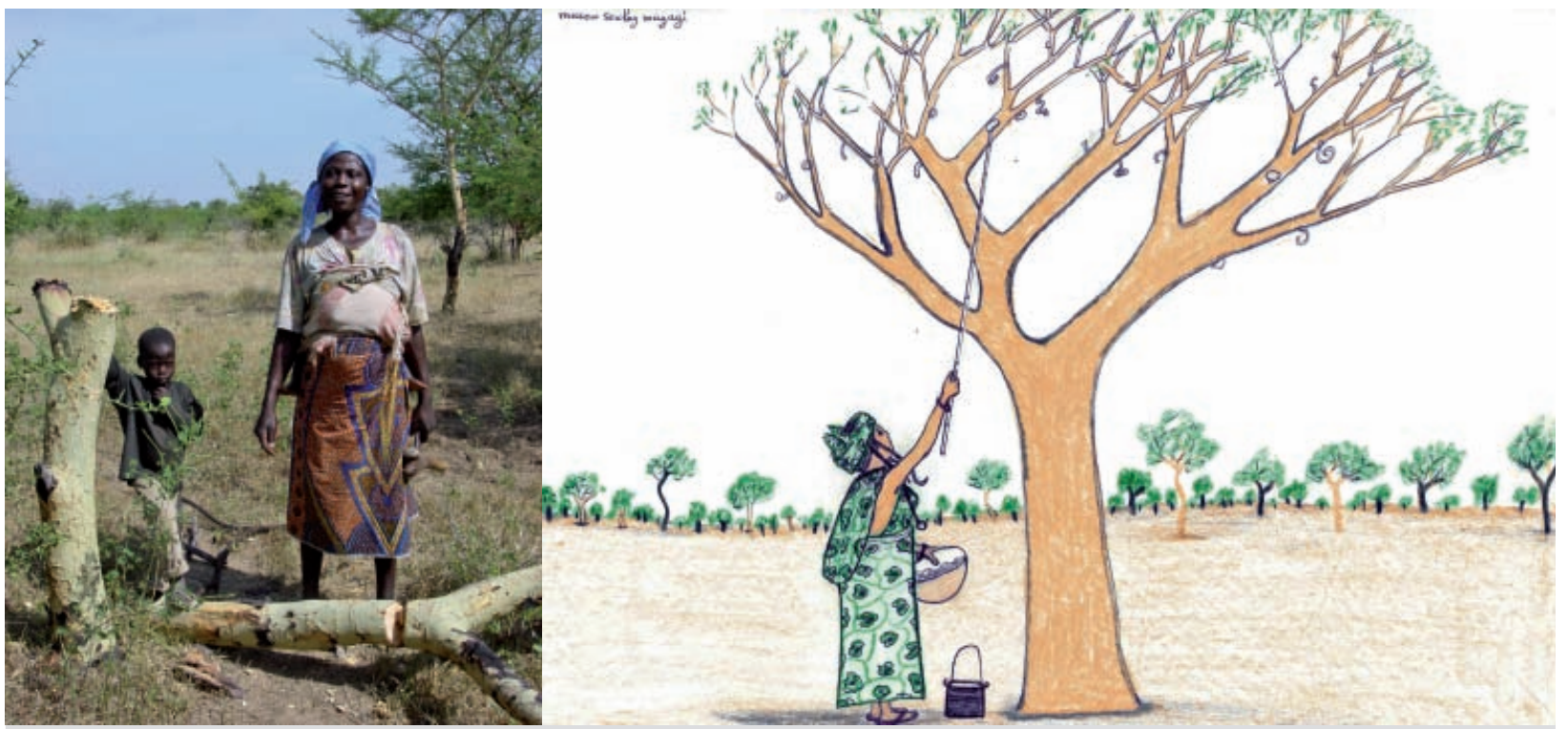

L'essentiel des quantités de gomme prélevées provient des formations naturelles à Acacia seyal. La récolte est effectuée en majorité par des enfants et des femmes profitant d'une autre activité en brousse pour compléter leurs revenus. Photo R. Peltier, dessin M.S. Maggi. 


\section{Discussion}

\section{Représentation des performances des filières par les acteurs}

La filière illégale présente des avantages pour les vendeurs, tels que le préfinancement de la campagne, le paiement au comptant, la multiplication des points d'achat jusque dans les villages et sur les marchés hebdomadaires, ce qui permet une réutilisation immédiate et sans risque de l'argent ainsi qu'une garantie d'achat, même si le prix est fluctuant et négociable.

Au contraire, la filière officielle est perçue comme rigide, les points d'achat sont considérés comme peu nombreux et éloignés des lieux de vente ; les prix sont fixes, en fonction de la qualité, mais la détermination de celle-ci est jugée arbitraire par les vendeurs ; les achats et les paiements ne sont pas garantis. S'agissant des prix, la filière officielle rémunère mieux la gomme dure triée d'Acacia senegal, qui est très rare : mais la filière illégale semble plus généreuse pour le mélange de gommes dures et friables, en particulier d'Acacia seyal, qui constitue l'énorme majorité des récoltes.

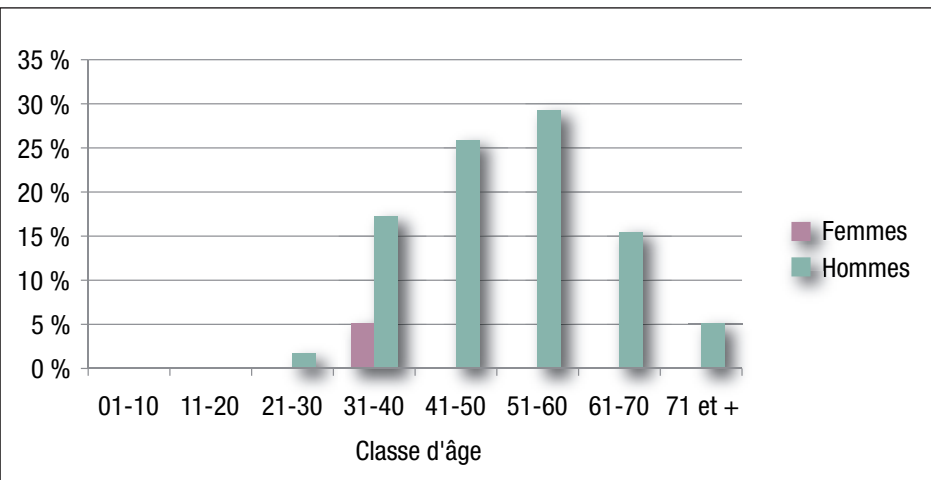

Figure 8.

Répartition des planteurs camerounais d'Acacia senegal par classe d'âge et par genre, en 2008.

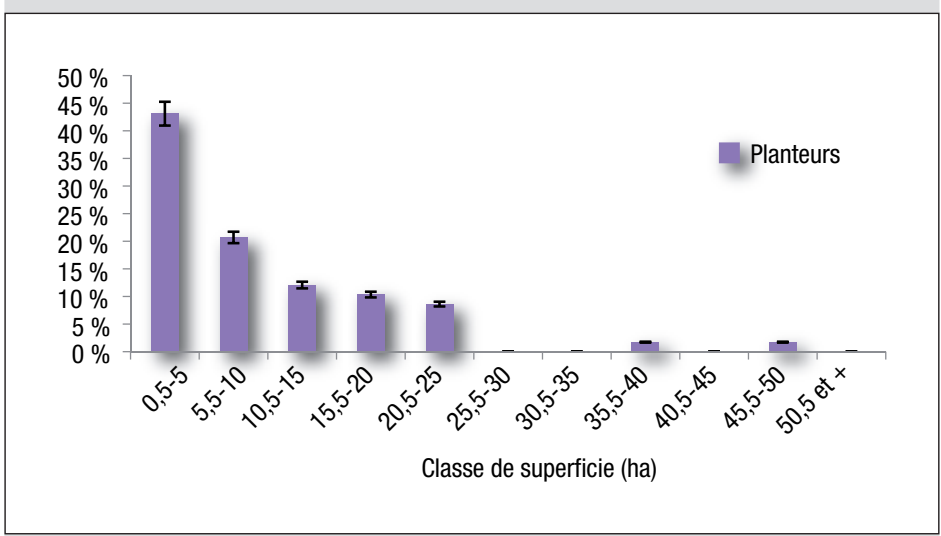

Figure 9.

Répartition des planteurs camerounais d'Acacia senegal selon la superficie des terres agricoles exploitées.

\section{Quantités et lieux de prélèvement}

Les résultats obtenus au Cameroun confirment ceux des études précédentes, en particulier de NјомAнA (2008). Cet auteur estimait la production globale de la gomme arabique au Nord-Cameroun à $1000 \mathrm{t} / \mathrm{an}$, parmi lesquelles 400 à 600 t vendues illégalement aux commerçants nigérians et 300 à 400 t achetées national par la société camerounaise d'exportation Cexpro. Il constatait que, sur la production globale, 95 à 99 \% provenait du grand peuplement naturel d'acacias lié au parc de Waza et que les plantations d'acacias ne fournissaient que 300 à 400 kg/an. Dans l'Est du Niger, les résultats de DuHEm (2004) montrent que les lieux de prélèvement de la gomme sont les formations naturelles à acacias (70\%), les jachères (15\%), les champs (10\%) et les plantations réalisées par des projets (4\%). II est donc retrouvé, comme au Cameroun, que les plantations jouent un rôle négligeable dans la production de gomme, par rapport aux formations naturelles. Au Niger, il faut cependant noter l'importance des recrûs naturels d'acacias dans l'espace agricole, champs ou jachères, qui pourraient constituer une première étape de domestication paysanne des gommiers, pas encore décrite au Cameroun.

\section{Types de gomme et de saignée}

Au Cameroun, la gomme friable d'Acacia seyal est la plus répandue et la plupart des cueilleurs ne trient pas la gomme ; il en est de même dans le Sud-Est du Tchad (VARDON, 1998). Dans l'Est du Niger, au contraire, la gomme est principalement prélevée sur Acacia senegal, secondairement sur $A$. tortilis ssp. raddiana et $A$. seyal, et les deux tiers des cueilleurs trient la gomme par espèce (DUHEM, 2004).

$\mathrm{Au}$ Cameroun, quasiment aucun cueilleur ne pratique la saignée dans les formations naturelles, il en est de même au Kenya (CHRETIN et al., 2007) ; au Niger, elle n'est pratiquée que par une minorité (DuHEM, 2004).

\section{Prix payé au cueilleur}

Au Cameroun, en 2008, le prix moyen annuel au niveau cueilleur est de $260 \mathrm{FCFA} / \mathrm{kg}$; il est supérieur à celui trouvé par BALARABÉ en 2000 (200 FCFA/kg) et NJOMAHA en 2008 (100-150 FCFA $/ \mathrm{kg})$. Il faut remarquer que, dans les pays voisins, le prix payé aux cueilleurs est également très bas. En mars 2004, dans l'Est du Niger, DuHEM (2004) notait que le prix de vente de la gomme dure était de $300 \mathrm{FCFA} / \mathrm{kg}$. Dans l'Est du Tchad (Salamat), VARDON (1998) notait le prix de $150 \mathrm{FCFA} / \mathrm{kg}$ payé aux cueilleurs pour la gomme friable "talha », qui est récoltée dans les vastes peuplements d'Acacia seyal, autour du parc national de Zacouma.

Même s'il est connu que les prix peuvent fluctuer de façon importante d'une année sur l'autre, selon les tensions du marché, il faut remarquer le grand écart entre le prix réellement payé au cueilleur $(0,2$ à $0,5 \$ \mathrm{US} / \mathrm{kg})$ et celui du marché international (1,5 à 4,5 $\$$ US/kg). Ces prix demandent cependant à être vérifiés au cours des prochaines cam- 


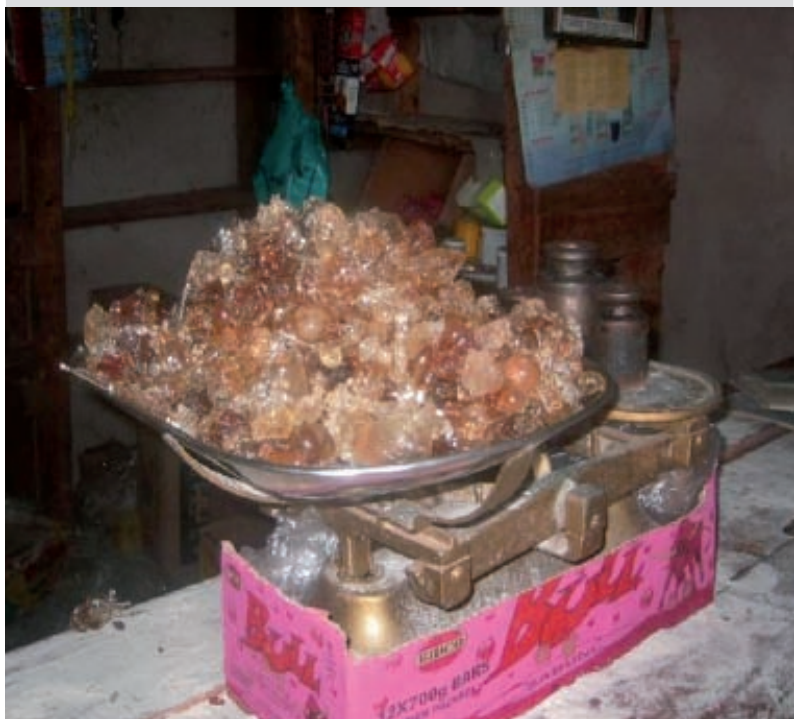

Près de $62 \%$ des collecteurs interrogés ne font pas de catégorisation de la gomme lors la collecte. Photo O. Palou Madi.

pagnes, d'autant plus que, depuis le début 2009, l'intervention de quelques acheteurs camerounais sans permis risque de perturber l'ancienne répartition tacite du marché, en introduisant une certaine concurrence.

Il faut cependant noter que les acheteurs de toute la région ont pris l'habitude, depuis plusieurs générations, d'acheter un mélange de gomme, à un prix très bas, qu'ils trient eux-mêmes et valorisent au mieux de leurs intérêts ; ils semblent faire très peu d'efforts pour mettre en place des filières de gomme triée ou pure, mieux payée au producteur.

\section{Détermination du prix d'achat par l'aval de la filière}

Au niveau officiel du Cameroun, l'existence d'un permis d'exportation délivré à un seul exportateur induit une organisation du commerce de la gomme en réseau et élimine un mode de marchandage purement libéral.

Les pratiques des opérateurs nigérians, avec leurs réseaux d'approvisionnement fidélisés et des liens de quasi-exclusivité du produit sur certaines zones frontalières, confortent davantage ce type d'organisation du marché de la gomme arabique. Une concordance des prix dans les étapes successives des circuits traduit une assez bonne circulation de l'information dans la filière, assurée par le déplacement simultané des commerçants et des collecteurs des villages de production vers les marchés (BALARABÉ, 2000).

\section{Composition des cueilleurs camerounais}

Il s'agit en majorité de femmes et de $10 \%$ d'enfants. Cette composition est comparable à celle du Niger, où ce sont les enfants qui sont majoritaires (près de $50 \%$ ) par rapport aux femmes (34\%).
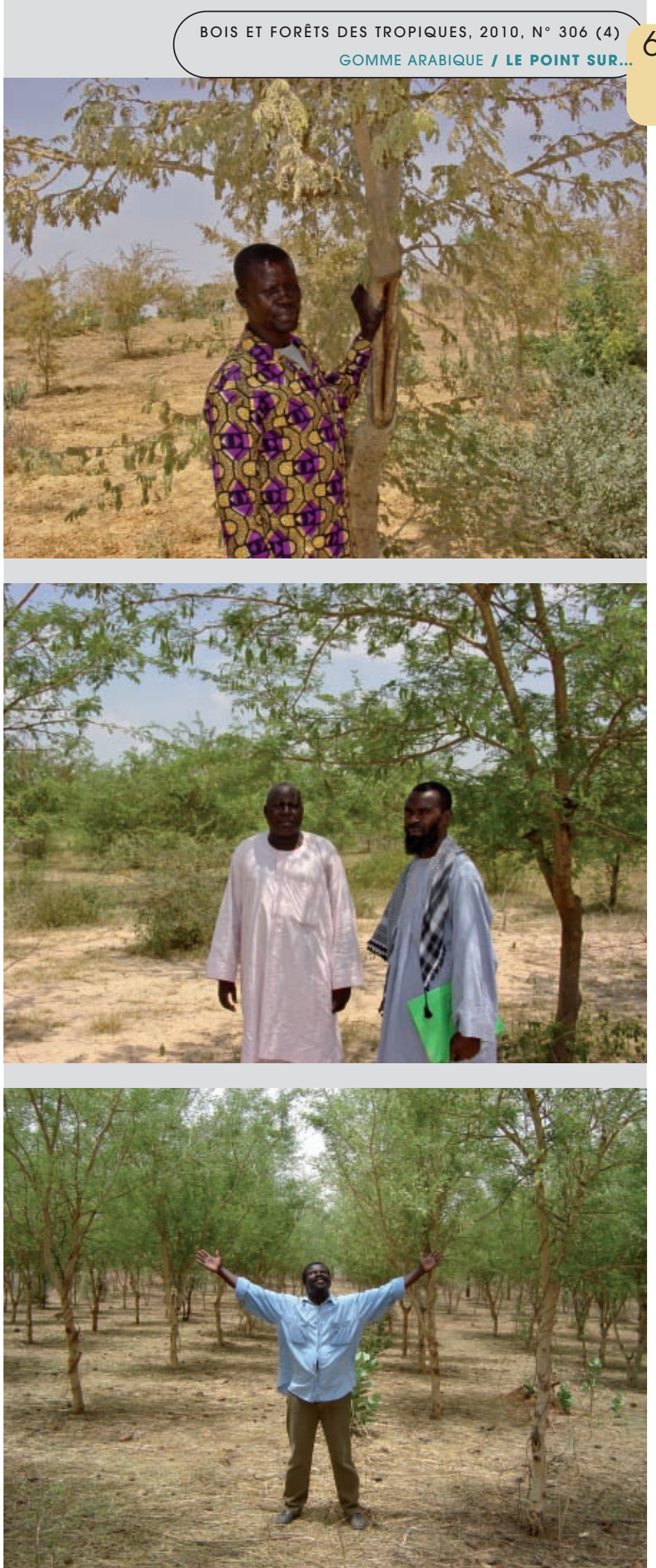

Les planteurs d'Acacia senegal camerounais constituent une population très différente de celle des cueilleurs. Il s'agit en majorité d'hommes d'âge mûr et relativement aisés.

Photos R. Peltier. 


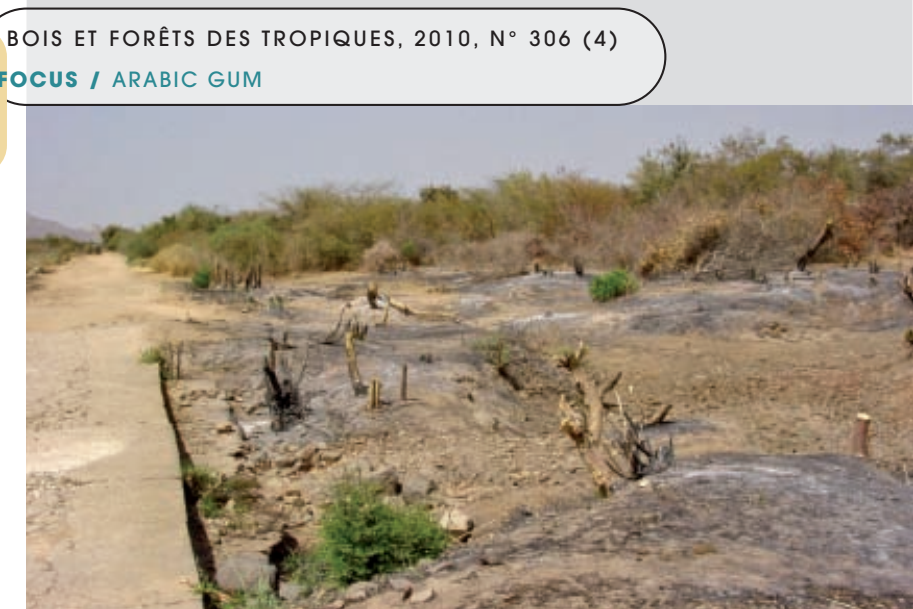

Il est estimé que les défrichements agricoles et la dégradation des formations naturelles finiront par tarir la filière cueillette de la gomme à l'horizon de plusieurs décennies. Photo R. Peltier.

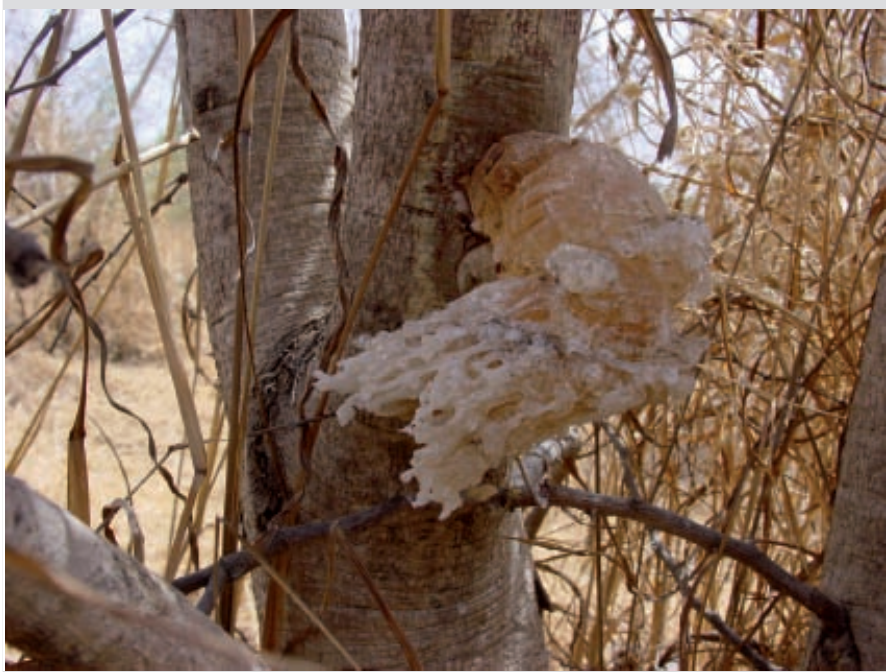

Les États ou les projets peuvent encourager les " gommiculteurs » à se positionner sur des marchés plus rémunérateurs, de type commerce équitable ou biologique. Photo R. Peltier.

\section{Population des planteurs de gommier camerounais}

Ils constituent une population très différente de celle des cueilleurs. Il s'agit en majorité d'hommes d'âge mûr, relativement aisés, natifs locaux qui possèdent de grandes surfaces et qui ont un revenu monétaire annuel non négligeable, par la vente du coton, des céréales et du bétail. Pour eux, la récolte de gomme n'est pas vitale et c'est pourquoi, contrairement aux cueilleurs, ils ne sont pas prêts à la vendre à vil prix ou à effectuer des dizaines de kilomètres pour la vendre sur les marchés.

Les projets et les Ong $^{15}$ qui ont encouragé les cueilleurs à s'organiser ou les agriculteurs à planter les ont motivés en leur promettant des prix élevés et une vente garantie dans le circuit officiel. Or, ils se sont souvent heurtés à la mévente de leur stock de gomme, qui n’intéressait pas beaucoup les acheteurs, en raison des faibles quantités, de leur dispersion et de l'impossibilité de jouer sur le poids et le prix, si bien que les organisations se sont dissoutes et que les plantations ont été abandonnées.
Il faut noter que la présente enquête n'a pas touché les agriculteurs qui n'ont pas actuellement d'activités en matière de plantation de gommiers mais qui pourraient être potentiellement intéressés. Les études antérieures (TEYSSIER, 2003 ; NJOMAHA, 2008) ont montré que de nombreux petits agriculteurs pourraient introduire un certain nombre de gommiers sur leur exploitation, au moins sous forme de haies vives ou de jachères enrichies, s'ils étaient certains de pouvoir garder les parcelles qu'ils auraient ainsi valorisées (sécurité foncière).

\section{Difficile passage de la cueillette de la gomme sauvage à la domestication du gommier}

Pour ce qui concerne la gomme au Nord-Cameroun, il est retrouvé une situation très courante dans le monde du développement, où des techniciens essayent d'aider des populations à passer de la cueillette à la production par ce qu'il est convenu d'appeler la « domestication » d'espèces végétales ou animales, voire d'écosystèmes. Il existe de nombreux exemples où des réussites techniques de ce type ne sont pas passées en milieu rural : plantation d'arbres producteurs de bois de feu en milieu rural au Sahel, élevage du petit gibier (aulacodes) en Afrique centrale, pisciculture en cage des tilapias au Niger et en Côte d'Ivoire, etc.

Ces mêmes techniques, par ailleurs « mécaniquement » au point, ne se sont diffusées que lorsque les prélèvements en milieu naturel n'étaient plus en mesure d'alimenter tout le marché (plantation de bois de feu au Burundi, pisciculture en cage au Viêt-Nam, etc.), lorsque le marché a exigé des produits normalisés (plantation de bois d'œuvre au Congo ou en Asie) ou lorsque les prix se sont envolés et que le marché a exigé des appellations d'origine contrôlée. Pour ce qui concerne la gomme, il peut être estimé que les défrichements agricoles (FotsIng, MAINAM, 2003) et la dégradation des formations naturelles finiront par tarir la filière cueillette, mais à l'horizon de plusieurs décennies. À court terme, seules l'augmentation du prix et la garantie d'achat pourraient encourager les planteurs à se former et d'appliquer les bonnes pratiques de gestion. Compte tenu de ce qui est observé sur le beurre de karité, le café et le cacao en Afrique, cela pourrait passer par la création de filières spécialisées de type «biologique ", « équitable » ou « éthique ». Cela est cohérent avec les propositions de MULLER (1995 et 1997), qui estimait que, pour une bonne perspective du marché, une prise en compte des préoccupations majeures des industriels utilisateurs de gomme serait nécessaire. Ce serait un approvisionnement régulier, une réduction des frais de nettoyage du produit et un niveau de prix de la gomme dure dans une fourchette de 2500 à 3000 \$ US/t. Cela passerait alors par des actions de valorisation du produit de quatre ordres :

- information du grand public ;

- mention explicite de la gomme arabique dans tous les produits où elle est utilisée (au lieu du seul code E 414) ;

- développement de la mention agrobiologique de la gomme ;

- écocertification de la gomme arabique. 


\section{Conclusion}

La filière illégale de la gomme est une réalité qui ne peut pas être ignorée dans des pays où les frontières sont incontrôlables. Les filières officielles ne peuvent être performantes que si elles adoptent globalement les mêmes méthodes d'achat, en particulier la multiplication des points d'achat, le préfinancement partiel et le paiement au comptant.

Si l'État et les projets veulent encourager des initiatives de gestion durable des peuplements gommiers naturels ou de reboisement de terres dégradées à base d'acacias, ils doivent appuyer la sécurisation foncière des plantations, encourager la création de forêts communautaires (pour sortir l'activité de cueillette de l'illégalité), réduire les taxes et tracasseries de toutes sortes qui pénalisent les filières officielles et imposer un triage sérieux à la base permettant de distinguer les gommes arabiques dures et friables des autres gommes, de qualité et de valeurs moindres.

Ils devront également encourager les " gommiculteurs » à se positionner sur des marchés plus rémunérateurs, de type commerce équitable ou biologique (avec l'appui technique de l'État, des projets et des Ong). Cela est tout à fait possible pour ce produit dont un des usages principaux est celui d'épaississant alimentaire de bonne qualité, qui entre dans la fabrication de produits à haute valeur ajoutée. Mais ces filières ne pourront se mettre en place qu'avec l'appui de projets, sur une assez longue durée, et en impliquant certains acheteurs et industriels finaux.

Il serait ainsi possible de montrer que l'encadrement des filières de produits forestiers ligneux ou non ligneux est un des leviers les plus efficaces pour piloter les écosystèmes anthropisés.

\section{Remerciements}

Ce travail a été réalisé avec le soutien du projet Acaciagum (financement Union européenne), de l'Irad, du Prasac et du ministère français des Affaires étrangères et européennes. Les auteurs souhaitent dédier cet article à Didier Muller, forestier qui a consacré une part importante de sa carrière au développement des plantations paysannes de gommier et qui est décédé tragiquement pendant l'été 2010.
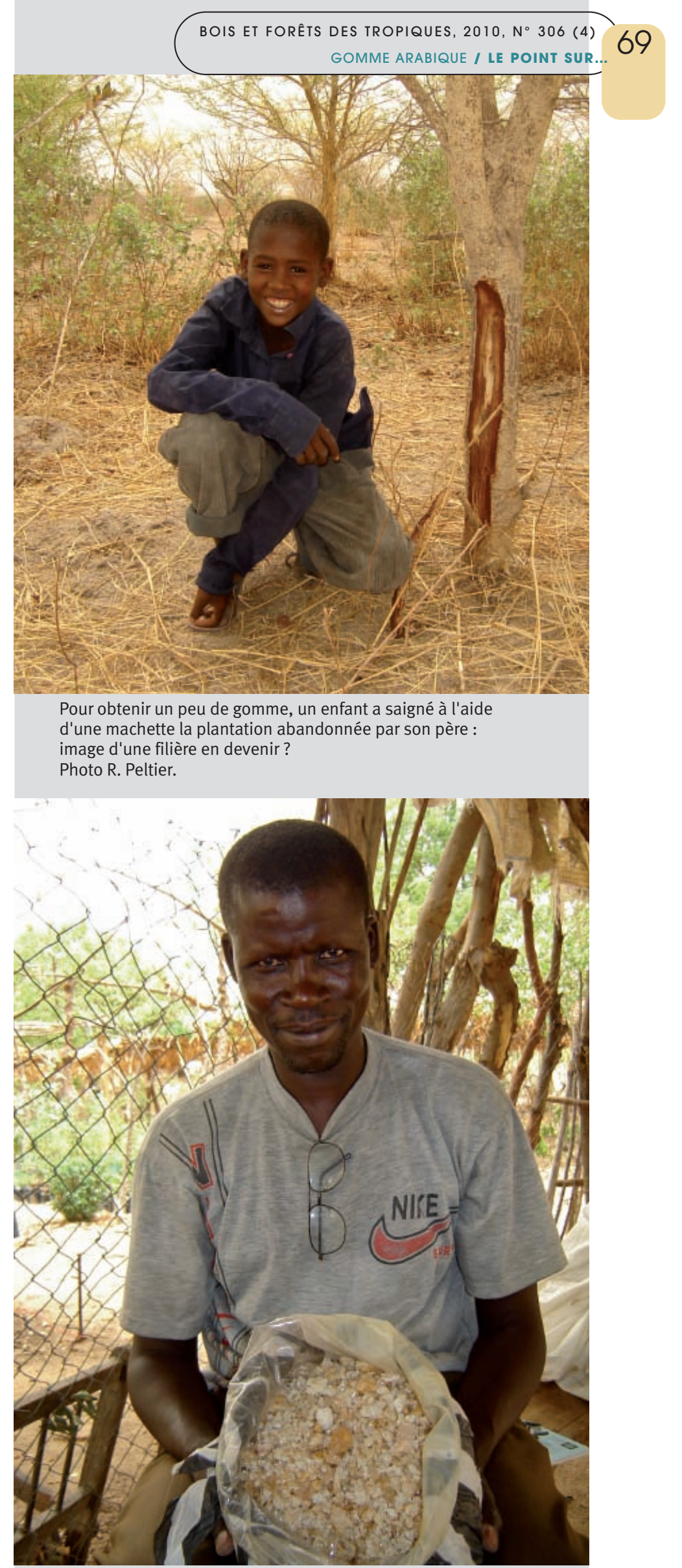

Un planteur du Pays Tupuri présente fièrement sa récolte annuelle de gomme arabique pure ; mais il devra la brader au prix de la gomme mélangée. Photo R. Peltier. 
ARBONNIER M., 2000. Arbres, arbustes et lianes des zones sèches d'Afrique de l'Ouest. Cirad-Mnhn-Uicn, 542 p.

BALARABÉ O., 2000. Filière gomme arabique dans le département du Logone et Chari. Fonctionnement et perspectives d'amélioration. Dschang, Cameroun, mémoire d’ingénieur, Fasa, 108 p.

BERGER A., LE COËNT P., 2001. Bilan des actions de promotion de l'arbre dans l'espace agraire effectuées par le projet Développement paysannal et gestion de terroir au NordCameroun : les actions de soutien aux pépiniéristes et de promotion de la jachère arborée. Montpellier, France, Engref, Cirad, Prasac, Irad et Ensa, 70 p.

CHRETIN M., CHIKAMAI B., EKAI LOKTARI P., NGICHILI J., LOUPA N., ODEE D., LESUEUR D., 2007. The current situation and prospects for Gum arabic in Kenya: a promising sector for pastoralists living in arid lands. International Forestry Review, 10 (1): 14-22.

DUHEM C., 2004. Étude des filières gomme dans les départements de Diffa et Maine Soroa. Méthodologie d'enquêtes, guides d'entretiens et questionnaires. Niamey, Niger, Pafn, 26 p.

DUGUÉ P., KOULANDJI J., MOUSSA C., 1994. Diversité et zonage des situations agricoles et pastorales de la zone cotonnière du Nord-Cameroun. Garoua, Cameroun, Ira-Irz, projet Garoua II, document de travail.

FOTSING E., MAINAM F., 2003. Dynamique du sorgho de contre saison et potentialités des sols en zone de savane de l'Extrême-Nord du Cameroun. In : Jamin J.-Y., Seiny Boukar L., Floret C. (éd.). Savanes africaines : des espaces en mutation, des acteurs face à de nouveaux défis. Actes du colloque, Garoua, Cameroun, 27-31 mai 2002, 7 p. http://hal.archives-ouvertes.fr/hal-00128923/fr/

HARMAND J.-M., MATHIEU B., NJITI C. F., NTOUPKA M., 1998. Recherche sur les possibilités de production de la gomme arabique par Acacia senegal (Linn.) Willd. dans différentes situations pédoclimatiques du Nord-Cameroun. Maroua, Cameroun, Irad, Projet de diversification des exportations agricoles au Cameroun, $21 \mathrm{p}$.

HARMAND J.-M., NJITI C. F., PELTIER R., 1997. Restauration de la fertilité des sols par la jachère arborée. Cycle de l'azote, statut organique du sol, production des cultures. In : L'agroforesterie pour un développement rural durable. Recherche fondamentale et modélisation, applications tempérées et méditerranéennes. Atelier international, Montpellier, France, 23-29 juin 1997. Montpellier, France, Cirad, p. 135-142.

MADI A., BALARABE O., TARLA F., 2002. Caractérisation de la filière gomme arabique au Nord-Cameroun. NgaoundéréAnthropos, vol. VII, 249 p.

MALLET B., BESSE F., GAUTIER D., MULLER D., BOUBA N., NJITI C., 2003. Quelles perspectives pour les gommiers en zone de savanes d'Afrique centrale ? In : Jamin J.-Y., Seiny Boukar L., Floret C. (éd.). Savanes africaines : des espaces en mutation, des acteurs face à de nouveaux défis. Actes du colloque, Garoua, Cameroun, 27-31 mai 2002. Montpellier, France, Cirad, cédérom.
MULLER D., 1995. La gomme arabique produit naturel de l'an 2000 ? Le Flamboyant, 34 : 14-18.

MULLER D., 1997. Acacias gommiers et gomme arabique : état des lieux et perspectives à la veille du troisième millénaire. Le Flamboyant, 43 : 26-31.

NJOMAHA C., 2008. Étude socio-économique de la filière gomme arabique dans le Nord et l'Extrême-Nord Cameroun. Rapport final. Maroua, Cameroun, Irad/Cedc-Snv, 125 p. + annexes.

PALOU MADI O., 2007. Introduction des acacias gommiers (Acacia senegal et $A$. polyacantha) en champs au NordCameroun : contraintes socio-économiques. Mémoire Master 2 Recherche "Essor », Université de Toulouse II, France, $74 \mathrm{p}$.

PELTIER R., 1993. Les jachères à composante ligneuse. Caractérisation, conditions de productivité, gestion. In : La jachère en Afrique de l'Ouest. Atelier international, Montpellier, France, 2-5 décembre 1991. Montpellier, France, Orstom, coll. Colloques et séminaires, p. 67-88.

PELTIER R., PALOU MADI O., BALARABE O., 2009. Les filières gomme arabique au Nord-Cameroun : impacts sur l'organisation des producteurs, la gestion des peuplements et l'encouragement des plantations. In : Actes du colloque « Savanes africaines en développement : innover pour durer ", Garoua, Cameroun, 20-24 avril 2009. http://hal.archivesouvertes.fr/docs/00/47/12/72/PDF/081_peltier.pdf

PRÉSIDENCE DE LA RÉPUBLIQUE DU CAMEROUN, 1994. Loi no 94/01 du 20 janvier 1994 portant régime des forêts, de la faune et de la pêche. Yaoundé, Cameroun, Présidence de la République, 24 p. http://www.droit-afrique.com/ images/textes/Cameroun/Cameroun\%20-\%20Loi\%20 foret.pdf

SODECOTON-DPA/ESA, 2006. Situation globale des réalisations d'action foresterie. Rapport d'activité, second semestre 2006. Garoua, Cameroun.

TEYSSIER A., 2003. La régulation foncière au Cameroun, entre régimes communautaires et aspirations citoyennes. In : Territoires ruraux, Actes du colloque, 25-27 février 2003. Montpellier, France, Cirad-Tera, 9 p.

TRIBOULET C., 1993. Évaluation des états de surface à partir d’images Spot. In : Peltier R. (éd.). Les terres Hardé : caractérisation et réhabilitation dans le bassin du lac Tchad. Montpellier, France, Cirad-Forêt, p. 29-36.

VARDON P., 1998. La gomme arabique dans le Salamat: quelle place, quels enjeux ? Montpellier, France, EngrefAfvp, $81 \mathrm{p}$. 